\title{
LITIASIS DE OXALATO CÁLCICO MONOHIDRATO PAPILAR Y DE CAVIDAD: ESTUDIO COMPARATIVO DE FACTORES ETIOLÓGICOS.
}

\author{
Enrique Pieras Ayala, Félix Grases Freixedas', Antonia Costa Bauzál, Margarita Ramis Barcelól, \\ Pedro Pizá Reus y Mariano Ozonas Moragues.
}

Servicio de Urología. Hospital Universitario de Son Dureta. Palma de Mallorca, Baleares.

Laboratorio de Investigación en Litiasis Renal'. Instituto Universitario de Investigación en Ciencias de la Salud (IUNICS). Universidad de las Islas Baleares. España.

\begin{abstract}
Resumen.- OBJETIVO: La litiasis de oxalato cálcico monohidrato (COM) se divide en dos grupos según su estructura morfocristalina: papilares (punto de anclaje sobre una lesión en la papila renall, y de cavidad (formados en una cavidad con baja eficacia urodinámica). Las escasas diferencias entre la bioquímica urinaria de los formadores de COM con respecto a la población sana sugiere que otros factores no relacionados con la bioquímica urinaria lactividad profesional, hábitos dietéticos, enfermedades sistémicas) pueden estar relacionados con la calculogénesis. El objetivo de este trabajo es el estudio de estos factores, y su comparación en ambos grupos de COM (papilar y de cavidad).
\end{abstract}

MÉTODOS: Se estudian 40 pacientes con litiasis de COM $(20$ pacientes con COM papilar y 20 pacientes con COM de cavidad). Se realiza historia clínica

Enrique Pieras Ayala

Álvaro de Bazán 3, Esc. 5, Ático l

07014 Palma. Islas Baleares. (España)

32477apa@comb.es

Trabajo recibido: 19 de octubre 2005 lantecedentes familiares de litiasis; enfermedades asociadas como hipertensión arterial, diabetes mellitus, hiperuricemia, hipercolesterolemia, úlcera gastroduodenal; encuesta de alimentación para valorar consumo de fitatos; profesiones con riesgo de exposición a agentes tóxicos), bioquímica de orina de 24 horas, orina de 2 horas $(\mathrm{pH})$, bioquímica plasmática. El estudio estadístico se realiza utilizando la $t$ de Student y la $X^{2}$.

RESULTADOS: Existe una elevada prevalencia de antecedentes familiares de litiasis renal (45\%) sin diferencias entre ambos grupos. No existen diferencias en la bioquímica urinaria ni plasmática. Existe un bajo consumo de alimentos que contienen fitatos en ambos grupos, sin existir diferencias significativas. Existe una tendencia hacia una mayor exposición a agentes citotóxicos en el grupo papilar (45\%) frente a cavidad (25\%). La hipertensión arterial e hiperuricemia son más prevalentes en el COM cavidad $/ \alpha=0,025$ y $\alpha=0,010$; respectivamente). La úlcera gastroduodenal es más prevalente en el COM papilar $(\alpha=0,025)$. No existen diferencias significativas en la prevalencia de hipercolesterolemia y diabetes mellitus entre ambos grupos.

CONCLUSIONES: Los cálculos COM papilares se asocian un déficit de inhibidores de la cristalización (fitatos), y a alteraciones del epitelio que recubre la papila renal lexposición a agentes citotóxicos, úlcera gastroduodenal). Los cálculos COM de cavidad se asocian a un déficit de inhibidores de la cristalización (fitatos) y a una mayor presencia de nucleantes heterogéneos Imateria orgánica inducida por enfermedades como la hipertensión arterial, hiperuricemia, hiperglucemia e hipercolesterolemia).

Palabras clave: Litiasis renal. Oxalato cálcico monohidratopapilar. Oxalato cálcico monohidrato de cavidad. Factores etiológicos. 
Summary.- OBJECTIVES: The monohydrate calcium oxalate lithiasis (MCO) is divided in two groups depending on the morphologic-crystal structure: papillary (anchorage point on a renal papilla lesion) and cavity (formed in a cavity with low urodynamic capacity). The minimal differences between urinary biochemistry of MCO makers in comparison with healthy population suggests that other factors different than urine biochemistry (professional activity, dietetic habits, systemic diseases) may be related with lithogenesis. The objective of this work is to study such factors, and compare them in both groups of MCO lithiasis (papillary and cavity).

METHODS: We study 40 patients with MCO lithiasis (20 patients papillary and 20 patients cavitary). Medical history was performed (family history of lithiasis; associated diseases such as high blood pressure, diabetes, hyperuricemia, hypercholesterolemia, peptic ulcer disease; dietetic survey to evaluate phytate consumption; professions with high-risk of exposure to toxic agents); 24-hour urine biochemical tests, two-hour urine $(\mathrm{pH})$, and serum biochemical profile were performed. Statistical analysis was performed using student's t test and chi-square.

RESULTS: There is a high prevalence of family history of renal lithiasis (45\%) without differences between groups. There are not differences in urine or blood biochemical tests. There is a low consumption of phytate-containing foods in both groups, without significant differences. There is a trend to a greater exposure to cytotoxic agents in the papillary group (45\%) vs. the cavity group (25\%). Hypertension and hyperuricemia are more prevalent in the cavity MCO group $/ \alpha=0.025$ and $\alpha=0.010$, respectively). Peptic ulcer disease is more prevalent in the papillary MCO group ( $\alpha=2.025$ ). There are no significant differences in prevalence of hypercholesterolemia or diabetes mellitus between groups.

CONCLUSIONS: Papillary MCO calculi are associated with a deficit of crystallization inhibitors (phytates), and disorders of the epithelium covering the renal papilla (cytotoxic agents exposure, peptic ulcer disease). Cavity MCO calculi are associated with a deficit of crystallization inhibitors (phytates) and a greater amount of heterogeneous nucleants /organic material induced by diseases such as hypertension, hyperuricemia, hyperglycemia, and hypercholesterolemial.

Keywords: Renal lithiasis. Papillary monohydrate calcium oxalate. Cavitary monohydrate calcium oxalate. Etiological factors.

\section{INTRODUCCIÓN}

La litiasis más frecuente es la de oxalato cálcico (63\%), pudiéndose dividir en dos grupos, oxalato cálcico dihidrato (COD) y oxalato cálcico monohidrato (COM). Sus alteraciones bioquímicas urinarias son distintas; en el COD se observa una excreción elevada de calcio en orina y una tendencia hacia un $\mathrm{pH}>6$, y en el COM existe una excreción normal de calcio en orina con un déficit de inhibidores de la cristalización (1 y 2).

Los cálculos de COM pueden dividirse a su vez en dos grupos según su morfología y estructura cristalina: COM papilar y COM no papilar o de cavidad. El COM papilar tiene una forma semiesférica, con una superficie cóncava que sirve de anclaje a la papila renal, con un corazón o zona de inicio cercano a esta zona, y desde aquí se distribuyen de forma radial y concéntrica las capas de cristales. El COM de cavidad se forma en una cavidad renal, tiene forma redondeada, sin puntos de anclaje a la papila renal, con un corazón claramente distinguible en situación central, que sirve de zona de inicio para la formación de cristales que constituirán el resto del cálculo (Figura $1 A B$, Figura $2 A B$ ).

Las diferencias morfológicas entre el COM papilar y COM de cavidad sugieren un mecanismo de formación distinto. En el caso del COM papilar el factor etiopatogénico principal es una lesión sobre la papila renal, que sirve de depósito para nucleantes heterogéneos, que a su vez inducen la formación de cristales; en el caso del COM de cavidad, la existen-

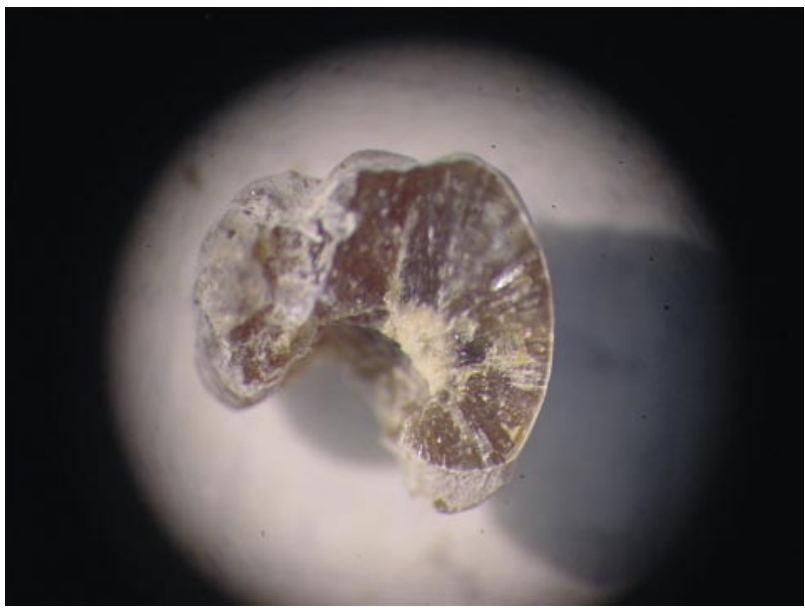

FIGURA IA. Imagen obtenida con microscopía binocular de cálculo de oxalato cálcico monohidrato papilar típico (corte transversal). 


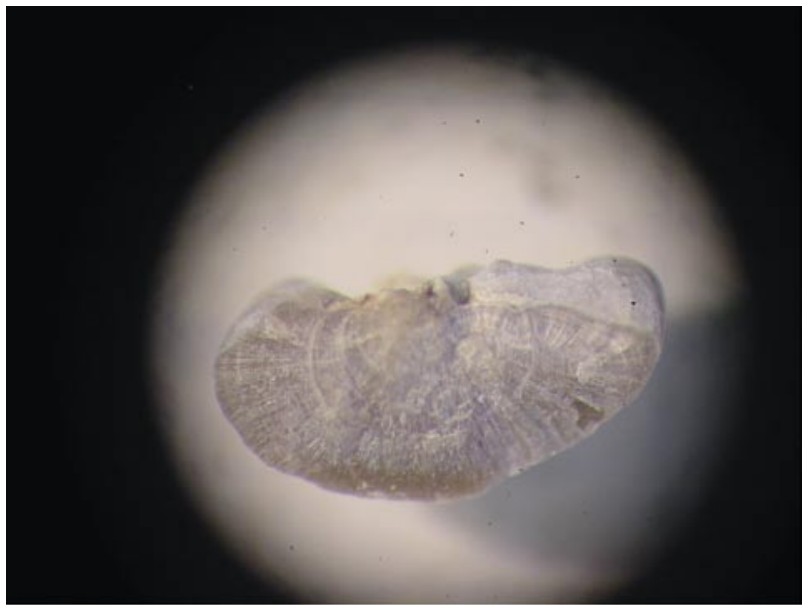

FIGURA 1B. Imagen obtenida con microscopía binocular de cálculo de oxalato cálcico monohidrato papilar típico (corte transversal).

cia de cavidades con baja eficacia urodinámica condicionan el depósito de nucleantes heterogéneos que favorecerán el desarrollo del cálculo. Por otra parte, no existen diferencias en la bioquímica urinaria de formadores de cálculos de COM con respecto a la población sana sin litiasis renal, lo que sugiere que otros factores no directamente relacionados con la bioquímica urinaria deben jugar un papel importante en la formación de estos cálculos (3).

El objetivo del presente estudio es conocer y comparar posibles factores etiológicos en ambos grupos (COM papilar y COM de cavidad) para entender mejor su mecanismo de formación, y por lo tanto, adoptar estrategias terapeúticas más adecuadas en cada grupo.

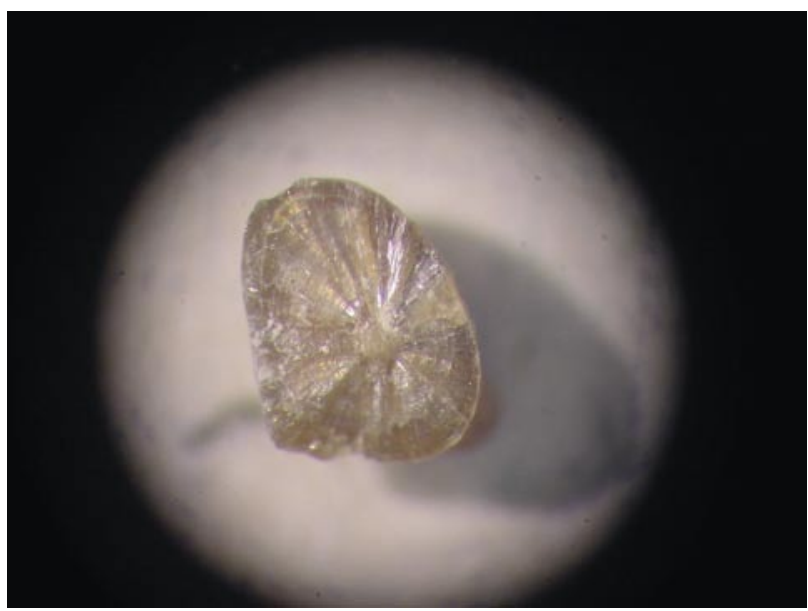

\section{PACIENTES Y MÉTODOS}

\section{Pacientes}

Estudiamos 40 pacientes formadores de cálculos de COM (20 exclusivamente COM papilar y 20 COM de cavidad). Ambos grupos tienen la misma distribución de sexo y edad. En todos ellos se realiza la historia clínica, se recogen sus hábitos dietéticos, su actividad profesional, sus antecedentes familiares de litiasis renal, y se analiza una muestra de orina de 24 horas y orina de 2 horas.

\section{Estudio de los cálculos}

Una vez que los cálculos son recogidos, se secan y se guardan en tubos estériles para su posterior estudio.

El procedimiento utilizado para el análisis y estudio del cálculo requiere una combinación de técnicas macroscópicas y microscópicas convencionales (microscopio estereoscópico Optomic) con técnicas físicas como la espectometría infrarroja (espectroscopio infrarrojo Brucker IFS 66) y microscopía electrónica con microanálisis por rayos $X$ (Hitachi S-530 de barrido con microanálisis por rayos $X$ Link Isis) (4).

\section{Estudio metabólico- bioquímica de la orina}

Todos los pacientes estaban con dieta libre en el momento de recoger la orina. Las determinaciones de creatinina, calcio, magnesio, fósforo, ácido úrico y colesterol total en plasma, se realizan para excluir los pacientes con insuficiencia renal. Los pacientes con orina infectada también fueron excluidos del estudio. La orina de 24 horas se recoge en un re-

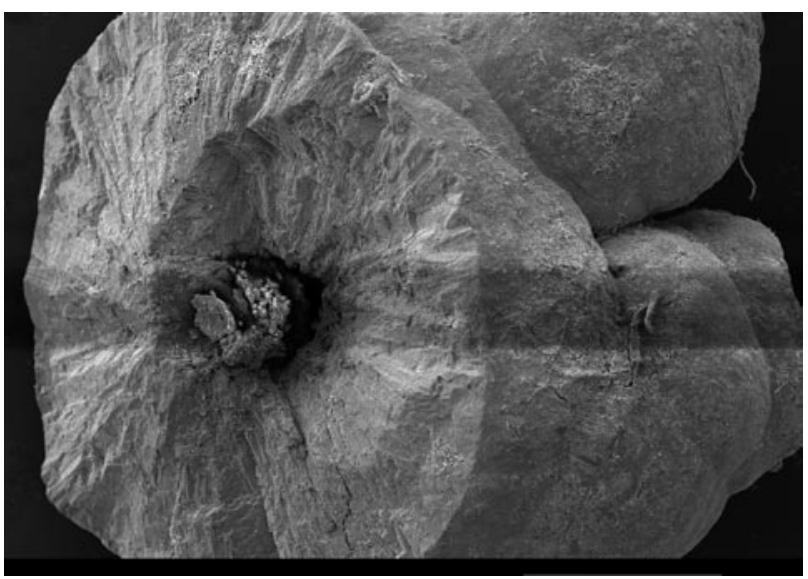

FIGURA 2. Imagen de cálculos de oxalato cálcico monohidrato de cavidad obtenida con microscopia binocular (izquierda), y microscopía electrónica de barrido en corte transversal (derecha). 
cipiente estéril con timol, se registra su volumen total, siendo inmediatamente refrigerada y conservada a - $20{ }^{\circ} \mathrm{C}$ hasta su análisis. La recogida de la primera muestra de orina de la mañana de 2 horas se realiza con el fin de determinar el $\mathrm{pH}$ urinario con un $\mathrm{pH}$ metro con electrodo de vidrio (Crison). En todos los casos la orina se recoge 1-2 meses después de la expulsión del cálculo.

El calcio, magnesio y fósforo se determinan mediante espectroscopia de emisión atómica con plasma acoplado inductivamente. El ácido úrico se determina con el método de la uricasa, por análisis colorimétrico enzimático en autoanalizador Hitachi, modelos 705 y 717 con reactivo de Boehringer Mannheim (cat. $n^{\circ} 908240 ; 1040936$ ). El citrato se determina con análisis enzimático UV en espectrofotómetro ultravioleta visible Perkin-Elmer mod. 552 con reactivo Boehringer Mannheim (cat. $n ¹ 39076$ ). La determinación de oxalato se realiza mediante análisis enzimático UV en espectofotómetro ultravioleta visible Perkin-Elmer mod. 552 con reactivo de Boehringer Mannheim (cat. $\mathrm{n}^{\circ}$ 755699). La creatinina se determina mediante el método de Jaffé cinético sin desproteinización en autoanalizador Hitachi, modelos 705 y 717 con reactivo de Sigma no 557 .

Todos los parámetros bioquímicos urinarios analizados se consideran como factores potenciales de riesgo litógeno cuando presentan un valor anormal de acuerdo con nuestra propia experiencia y la de la literatura, definidos en la Tabla I.

TABLA I. VALORES DE RIESGO METABÓLICO LITÓGENO EN LA BIOQUIMMICA DE ORINA 24 HORAS Y ORINA DE 2 HORAS (PH).

\begin{tabular}{|l|l|}
\hline $\mathrm{pH}$ & $<5.5$ \\
& $>6.0$ \\
\hline Creatinina $(\mathrm{mg} / 24 \mathrm{~h})$ & $>2000$ \\
\hline $\mathrm{Ca}(\mathrm{mg} / 24 \mathrm{~h})$ & $>250$ (mujer) \\
& $>300$ (hombre) \\
\hline $\mathrm{Mg}(\mathrm{mg} / 24 \mathrm{~h})$ & $<70$ \\
\hline $\mathrm{P}(\mathrm{mg} / 24 \mathrm{~h})$ & $>1200$ \\
\hline Oxalato $(\mathrm{mg} / 24 \mathrm{~h})$ & $>40$ \\
\hline Acido úrico $(\mathrm{mg} / 24 \mathrm{~h})$ & $>600$ (mujer) \\
& $>800$ (hombre) \\
\hline Citrato $(\mathrm{mg} / 24 \mathrm{~h})$ & $<350$ \\
\hline
\end{tabular}

Actividad profesional, antecedentes familiares de litiasis renal, hábitos dietéticos y enfermedades asociadas.

Para todos los pacientes se obtiene la historia clínica de enfermedades asociadas (hipertensión arterial, hipercolesterolemia, hiperuricemia, diabetes mellitus, sobrepeso, úlcera gastroduodenal, stress, colelitiasis, cáncer, enfermedades vasculares y enfermedades gastrointestinales). Se realiza un cuestionario de hábitos dietéticos, con especial interés en alimentos que contienen fitatos (alimentos integrales, fibra dietética, frutos secos y legumbres). Se recogen los antecedentes familiares de litiasis renal y la actividad profesional, en especial las profesiones con riesgo de exposición a agentes citotóxicos (pesticidas, herbicidas, productos de limpieza y desinfección, agentes carburantes y productos utilizados en la industria química).

\section{Estudio estadístico}

En el tratamiento estadístico se utiliza la t de Student para variables cuantitativas y la $X^{2}$ (chi-cuadrado) con el estadístico de contrate $\lambda$ de Pearson para variables cualitativas.

\section{RESULTADOS}

Los datos analíticos de la bioquímica de orina de 24 horas y de 2 horas se muestran en la tabla II. Como puede observarse, no se aprecian diferencias estadísticamente significativas entres ambos grupos en ninguno de los parámetros analizados. Sin embargo, si observamos las desviaciones estándar de la tabla II puede apreciarse que existen algunos pacientes con hipocitraturia, hipercalciuria, hiperuricuria o alteraciones en el pH. (Tabla II).

Se observa una prevalencia del $45 \%$ de antecedentes familiares de litiasis renal, sin que existan diferencias estadísticamente significativas entre ambos grupos. Un $50 \%$ y $40 \%$ de los COM de cavidad y papilar tienen antecedentes familiares previos, respectivamente.

Al analizar los hábitos dietéticos observamos como existe un bajo consumo de alimentos ricos en fitatos en ambos grupos, aunque sin que existan diferencias estadísticamente significativas entre ellos (Tabla III).

Hemos encontrado una elevada prevalencia de profesiones con riesgo de exposición a agentes citotóxicos, 14 de 40 (35\%): 6 agricultores (herbicidas y pesticidas), 3 obreros de la construcción (in- 


\section{TABLA II. VALORES DE PH URINARIO Y DE EXCRECIÓN DE LA BIOQUÍMICA URINARIA EN RELACIÓN CON EL TIPO DE CÁLCULO. (VALORES EXPRESADOS COMO MEDIA \pm DESVIACIÓN ESTÁNDARI.}

\begin{tabular}{|l|c|c|}
\hline Parámetros urinarios & Cálculos COM papilar $(\mathrm{n}=20)$ & Cálculos COM de cavidad $(\mathrm{n}=20)$ \\
\hline $\mathrm{pH}$ & $5.7 \pm 0.6$ & $5.8 \pm 0.7$ \\
\hline Creatinina $(\mathrm{mg} / 24 \mathrm{~h})$ & $1373 \pm 444$ & $1180 \pm 425$ \\
\hline $\mathrm{Ca}(\mathrm{mg} / 24 \mathrm{~h})$ & $224 \pm 184$ & $206 \pm 121$ \\
\hline $\mathrm{Mg}(\mathrm{mg} / 24 \mathrm{~h})$ & $95 \pm 42$ & $84 \pm 42$ \\
\hline $\mathrm{P}(\mathrm{mg} / 24 \mathrm{~h})$ & $1047 \pm 565$ & $821 \pm 299$ \\
\hline Oxalato $(\mathrm{mg} / 24 \mathrm{~h})$ & $23.1 \pm 8.5$ & $19.6 \pm 9.8$ \\
\hline Acido úrico $(\mathrm{mg} / 24 \mathrm{~h})$ & $625 \pm 232$ & $582 \pm 236$ \\
\hline Citrato $(\mathrm{mg} / 24 \mathrm{~h})$ & $544 \pm 266$ & $611 \pm 286$ \\
\hline
\end{tabular}

halación del polvo de la obra), 3 trabajadores de la empresa de limpieza (productos de limpieza y desinfección), 1 trabajador de la industria química del caucho y 1 técnico de aviación (carburantes). Se observa una mayor prevalencia de exposición a sustancias citotóxicas en el grupo de COM papilar, 9 pacientes $(45 \%)$ frente a 5 pacientes $(25 \%)$ en el grupo de COM de cavidad.

En las enfermedades asociadas a ambos grupos observamos una elevada prevalencia de enfermedades como la hipertensión arterial, hipercolesterolemia, diabetes mellitus e hiperuricemia, siendo la mayoría de ellas más prevalentes en el grupo de COM de cavidad. Se observa como la hipertensión arterial y la hiperuriemia son más prevalentes en el grupo de COM de cavidad con diferencias estadísticamente significativas $(\alpha=0,025$ y $\alpha=0,010$, respectivamente), y la úlcera gastroduodenal es significativamente más prevalente en el grupo de COM papilar $(\alpha=0,025)$. (Tabla IV y TablaV).

\section{DISCUSIÓN}

No hemos observado ninguna alteración bioquímica específica en la orina de los formadores de cálculos de COM (papilar y de cavidad), sin embargo, las altas desviaciones standard para el citrato, calcio y ácido úrico confirman que existen pacientes con alguna alteración, como la hipocitraturia, hipercalciuria e hiperuricosuria (Tabla II). No existen diferencias entre la orina de los formadores de COM con respecto a la población sana sin litiasis renal 3 y 5), por lo tanto era de esperar que tampoco en este estudio se encontraran diferencias en la bioquímica urinaria de los formadores de COM papilar y de cavidad.
Los pacientes con antecedentes familiares de litiasis renal presentan un riesgo mayor de padecer litiasis que el resto de la población (6). En nuestro estudio se observa una elevada prevalencia $(45 \%)$ de antecedentes familiares de litiasis renal, siendo un claro indicio de la posible influencia de factores genéticos en los dos grupos estudiados. Así, la morfología renal que puede relacionarse con unas cavidades con baja eficacia urodinámica, así como las mucosas poco protegidas con tendencia a sufrir lesiones epiteliales, son características que podrían ir ligadas a la herencia genética. Sin embargo, la herencia genética no puede por sí sola explicar el desarrollo de los cálculos, ya que la litiasis es una enfermedad multifactorial donde intervienen múltiples factores.

El fitato es un producto natural presente en los alimentos ricos en fibra (legumbres, frutos secos, cereales integrales) que constituye uno de los inhibidores de cristalización de sales cálcicas más efectivos (7). Existen estudios epidemiológicos que han puesto de manifiesto la relación existente entre el bajo consumo de fitatos y la litiasis renal (8 y 9). La cantidad de fitato en el organismo se encuentra directamente relacionado con su ingesta oral a través de distintos alimentos, especialmente en legumbres, pan integral, otros alimentos integrales, frutos secos y fibra dietética, sin que exista una producción del mismo de forma endógena (10). En nuestro estudio hemos encontrado un bajo consumo de alimentos ricos en fitato en ambos grupos, sin diferencias estadísticamente significativas (Tabla III). Estos hechos sugieren que la formación de cálculos de COM papilares y de cavidad está relacionado con un déficit de inhibidores de la cristalización.

Hemos observado una mayor prevalencia de actividades profesionales con riesgo de exposición a 
TABLA III. ESTUDIO DE HÁBITOS DIETÉTICOS: CONSUMO DE ALIMENTOS RICOS EN FITATO (LEGUMBRES, FIBRA DIETÉTICA, ALIMENTOS INTEGRALES Y FRUTOS SECOS).

\begin{tabular}{|c|c|c|}
\hline $\begin{array}{c}\text { Consumo de productos ricos } \\
\text { en fitato (veces por semana) }\end{array}$ & $\begin{array}{c}\text { Número de formadores de COM } \\
\text { papilar }(\mathrm{n}=20)\end{array}$ & $\begin{array}{c}\text { Número de formadores de COM no } \\
\text { papilar }(\mathrm{n}=20)\end{array}$ \\
\hline 0 & 0 & 1 \\
\hline 2 & 4 & 3 \\
\hline 4 & 7 & 6 \\
\hline 6 & 4 & 7 \\
\hline 8 & 3 & 3 \\
\hline 10 & 2 & 0 \\
\hline Consumo medio & 5.2 & 4.8 \\
\hline
\end{tabular}

* Para obtener unos niveles de fitato normales se considera un consume de alimentos ricos en fitato igual o superior a 7 (23).

agentes citotóxicos (pesticida, herbicidas, productos limpieza y desinfección,...) en el grupo de COM papilar. Estos resultados pueden justificarse debido al efecto citotóxico que estos productos provocan en el urotelio papilar, y en todos los epitelios en general, generando una lesión celular en la papila renal, que servirá de depósito para nucleantes heterogéneos, que inducirán el crecimiento de cristales de COM sobre su superficie, generando por lo tanto un cálculo papilar (11 y 12). De hecho, se ha demostrado claramente cómo el consumo de productos citotóxi- cos como el etilenglicol o el abuso de analgésicos generan calcificaciones papilares (13 y 14).

Al estudiar las enfermedades asociadas hemos encontrado diferencias estadísticamente significativas en varias de ellas, así la úlcera gastroduodenal se asocia con cálculos de COM papilares, y la hipertensión e hiperuricemia con cálculos de COM de cavidad. Esta asociación podría explicarse considerando que la úlcera está relacionada con una lesión del epitelio de la mucosa gastroduodenal, y los cálculos

\section{TABLA IV. RELACIÓN DE PREVALENCIA DE ENFERMEDADES ESTUDIADAS EN CADA GRUPO DE CÁLCULOS COM.}

\begin{tabular}{|l|c|c|}
\hline Enfermedades asociadas & $\begin{array}{c}\text { Número de formadores de COM } \\
\text { papilar }(\mathrm{n}=20)\end{array}$ & $\begin{array}{c}\text { Número de formadores de COM de } \\
\text { cavidad }(\mathrm{n}=20)\end{array}$ \\
\hline Cancer & 0 & 1 \\
\hline Diabetes Mellitus & 1 & 4 \\
\hline Enfermedades gastrointestinales & 2 & 1 \\
\hline Enfermedades vasculares & 1 & 2 \\
\hline Hipercolesterolemia & 7 & 10 \\
\hline Hiperuricemia & 4 & 12 \\
\hline Hipertensión arterial & 4 & 11 \\
\hline Colelitiasis & 2 & 2 \\
\hline Stress & 0 & 1 \\
\hline Sobrepeso & 2 & 4 \\
\hline Ulcera gastroduodenal & 5 & 0 \\
\hline
\end{tabular}




\section{COM cavidad $\square$ Com papilar}

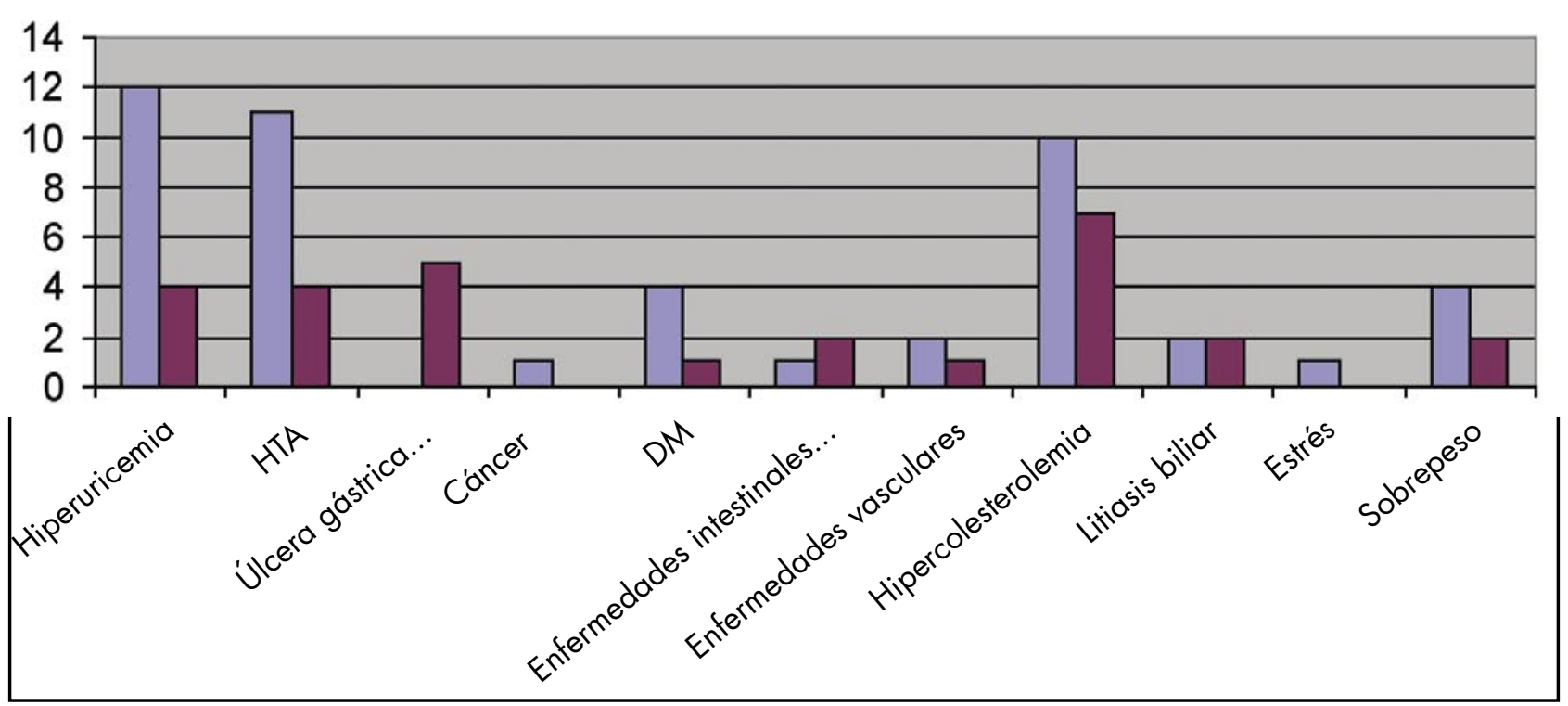

papilares con una lesión en el epitelio de la papila renal, por lo que compartirían un mecanismo etiopatogénico de lesión epitelial (16-19). Por otra parte, la hipertensión arterial y la hiperuriemia son más prevalentes en el grupo de COM de cavidad, esta diferencia podría ser debida a que ambas enfermedades se relacionan con mayor cantidad de materia orgánica en orina, que se comporta como un importante inductor (nucleante heterogéneo) de los cristales de COM y quedarían retenidos en cavidades con baja eficacia urodinámica $(17,20-22)$.

\section{CONCLUSIONES}

Los cálculos papilares de COM se asocian a un déficit de inhibidores de la cristalización (fitatos), y a alteraciones del epitelio de la papila renal, como lo demuestra una mayor prevalencia de exposición a agentes citotóxicos y de úlcera gastroduodenal.

Los cálculos de cavidad de COM se asocian a un déficit de inhibidores de la cristalización (fitatos), y a una mayor presencia de nucleantes heterogéneos en la orina, que son materia orgánica inducida por enfermedades más prevalentes en este grupo, como la hipertensión arterial y la hiperuricemia, y en menor grado, la hiperglucemia y la hipercolesterolemia.

\section{BIBLIOGRAFÍA y LECTURAS RECOMENDADAS ( ${ }^{*}$ lectura de interés $y^{* *}$ lectura fundamental)}

**1. GRASES, F.; COSTA-BAUZÁ, A.; RAMIS, M.: "Simple classification of renal calculi closely related to their micromorphology and etiology". Clinica Chimica Acta, 322: 29, 2002.

*2. GRASES, F.; CONTE, A.; COSTA-BAUZÁ, A.: "Tipos de cálculos renales. Relación con la bioquímica urinaria”. Arch. Esp. Urol., 54: 861, 2001.

3. GALÁN, J.A.; CONTE, A.; LlOBERA, A.: "A comparative study between etiological factors of calcium oxalate monohydrate and calcium oxalate dihydrate urolithiasis". Urol. Int., 56: 79, 1996.

4. GRASES, F.; GARCÍA-FERRAGUT, L.; COSTA-BAUZÁ, A.: "Analytical study of renal calculi. A new insight”. Recent Res. Dev. Pure Appl. Anal Chem., 1: 187, 1998.

5. PIERRATOS, A.E.; KHALAFF, H.; CHENG, P.T. y cols.: "Clinical and biochemical differences in patients with pure calcium oxalate monohydrate and calcium oxalate dihydrate kidney stones". J. Urol., 151: 571, 1994.

6. CURHAN, G.C.; WILLETT, W.C.; RIMM, E.B. y cols.: "Family history and risk of kidney stones”. J. Am. Soc. Nephrol., 8: 1568, 1997.

7. GRASES, F.; KROUPA, M.; COSTA-BAUZÁ, A.: "Studies on calcium oxalate monohydrate crystallization. Influence of inhibitors”. Urol. Res., 22: 39, 1994. 
**8. MODLIN, M.: "Urinary phosphorylated inositols and renal stone". Lancet, 2: 1113, 1980.

9. CURHAN, G.C.; WILLETT, W.C.; KNIGHT, E.L. y cols.: "Dietary factors and the risk of incident kidney stones in younger women: Nurses Health Study II". Arch. Intern. Med., 164: 885, 2004.

**10. GRASES, F.; SIMONET, B.M.; MARCH, J.G. y cols.: "Inositol hexakisphosphate in urine: the relationship between oral intake and urinary excretion". BJU Int., 85: 138, 2000.

11. GRASES, F.; GARCÍA-FERRAGUT, L.; COSTA-BAUZÁ, A.: "Development calcium oxalate crystals on urothelium: effect of free radicals". Nephron, 78: 296, 1998.

12. GRASES, F.; GARCÍA-FERRAGUT, L.; COSTA-BAUZÁ, A.: "Study of the early stages of renal stone formation. Experimental model using urothelium of pig urinary bladder". Urol. Res., 24: 305, 1996.

*13. THAMILSELVAN, S.; HACKETT, R.L.; KHAN, S.R.: "Lipid peroxidation in ethylene glycol induced hyperoxaluria and calcium oxalate nephrolithiasis". J. Urol., 157: 1059, 1997.

14. SCHWARZ, A.: "Bethoven's renal disease based on his autopsy: a case of papillary necrosis". Am. J. Kidney Dis., 21: 643, 1993.

15. TARNAWSKI, A.; SZABO, I.L.; HUSAIN, S.S. y cols.: "Regeneration of gastric mucosa during ulcer healing is triggered by growth factos and signal transduction pathways". J. Phsysiol. Paris, 95: 337, 2001.
16. DE WATER, R.; NOORDERMEER, C.; HOUSTMULLER, A.B. y cols.: "Role of macrophages in nephrolithiasis in rats: an analysis of the renal interstitium”. Am. J. Kidney Dis., 36: 615, 2000.

17. KHAN, S.R.; THAMILSELVAN, S.: "Nephrolithiasis: a consequence of renal epithelial cell exposure to oxalate and calcium oxalate crystals". Mol. Urol., 4: 305, 2000.

18. DE WATER, R.; NOORDERMEER, C.; VAN DER KWAST, T.H. y cols.: "Calcium oxalate nephrolithiasis: effect of renal crystal deposition on the cellular composition of the renal interstitium". Am. J. Kidney Dis., 33: 761, 1999.

19. MUTHUKUMAR, A.; SELVAM, R.: "Renal injury mediated calcium oxalate nephrolithiasis: role of lipid peroxidation". Ren. Fail., 19: 401, 1997.

*20. KHAN, S.R.: "Heterogenus nucleation of calcium oxalate crystals in mammalian urine". Scanning Microscopy, 9: 597, 1995.

**21. KHAN, S.R.; GLENTON, P.A.; BACKOV, R. y cols.: "Presence of lipids in urine, crystals and stones: implications for the formation of kidney stones". Kidney Int., 62: 2062, 2002.

22. KHAN, S.R.; MASLAMANI, S.A.; ATMANI, F. y cols.: "Membranes and their constituents as promoter of calcium oxalate crystal formation in human urine". Calcif Tissue Int., 66: 90, 2000.

23. GRASES, F.; SIMONET, B.M.; VUCENIK, I. y cols.: "Absorption and excretion of orally administered inositol hexaphosphate (IP6 or phytate) in humans". BioFactors, 15: 53, 2001. 